\section{$\underset{\substack{\text { hommes } \\ \text { \& migrations }}}{ }$}

\section{Hommes \& migrations}

Revue française de référence sur les dynamiques

migratoires

\section{$1279 \mid 2009$}

L'Afrique en mouvement

\title{
Le "gouvernement humanitaire"
}

Entretien réalisé par Marie Poinsot avec Michel Agier

\section{Marie Poinsot et Michel Agier}

\section{(2) OpenEdition}

1 Journals

\section{Édition électronique}

URL : http://journals.openedition.org/hommesmigrations/329

DOI : 10.4000/hommesmigrations.329

ISSN : 2262-3353

Éditeur

Musée national de l'histoire de l'immigration

\section{Édition imprimée}

Date de publication : 1 mai 2009

Pagination : 104-113

ISSN : 1142-852X

\section{Référence électronique}

Marie Poinsot et Michel Agier, "Le "gouvernement humanitaire" », Hommes \& migrations [En ligne], 1279 | 2009, mis en ligne le 29 mai 2013, consulté le 19 avril 2019. URL : http://

journals.openedition.org/hommesmigrations/329 ; DOI : 10.4000/hommesmigrations.329 


\section{Le "gouvemement humanitaire"}

Entretien réalisé par Marie Poinsot avec Michel Agier, anthropologue, directeur d'études à l'EHESS et directeur de recherche

à l'IRD, dirige depuis 2004 le Centre d'études africaines, une unité mixte de recherche IRD-EHESS

\section{H\&M : Comment avez-vous été amené à travailler sur les réfugiés en Afrique ?}

J'ai mené des recherches en Amérique latine et en Afrique, sur les zones urbaines précaires, les zones marginales, les quartiers spontanés (comme on le dit en Afrique) ou les invasions (Amérique latine), donc sur toutes les situations d'habitat et d'installation précaires dans les contextes urbains. C'est comme cela que je me suis intéressé à la question des déplacés/réfugiés en commençant en Amérique latine, et plus précisément en Colombie, où j'ai étudié les migrants qui, en 19981999, fuyaient de la côte pacifique vers la ville de Cali. Ces déplacés internes fuyaient les zones de conflit entre les paramilitaires et la guérilla.

En revenant de Colombie, par le biais d'un travail sur les réfugiés, j’ai démarré un programme de recherche en Afrique, consacré en particulier à la question des camps, puisque ma problématique est de savoir où et comment ces personnes trouvent leur place, dans une situation de déplacement, en fonction des différents contextes locaux (urbains, très urbains, ruraux, désertiques, dangereux, sécurisés, etc.). Il faut rappeler que l'exil est une pratique assez généralisée dans le monde, que ce soit du fait des guerres ou de situations sociales et économiques catastrophiques. Dans le cadre de cette recherche, je me suis intéressé au dispositif humanitaire, c'està-dire à l'ensemble de ce que crée l'intervention internationale des ONG auprès de populations en danger. Des systèmes de contrôle se mettent en place, d'où le nom que j'ai proposé de "gouvernement humanitaire", qui constitue désormais une nouvelle forme de gouvernement dans le monde, et en Afrique en particulier.

\section{H\&M : Vous avez étudié la réalité des réfugiés dans deux continents. Peut-on dire qu'il y a des particularités propres à l'Afrique ? À la fois dans le type de déplacement, les raisons ou les situations que ces migrations engendrent ? \\ Il est délicat de répondre sans être tautologique : "s'il y a des réfugiés c'est parce que ces personnes ont été définies comme réfugiés". La question est plutôt celle de savoir com-}


ment et qui classe ces réfugiés. On peut dire que les situations de guerre et de persécution créent de manière générale l'exil comme conséquence de l'impossibilité de vivre quelque part. Les situations sont toujours différentes et pourtant toujours égales, que ce soit l'exil des Arméniens dans les années vingt, des Espagnols dans les années trente ou des populations d'Europe pendant la Seconde Guerre mondiale, bref toutes ces vagues de réfugiés qui, par arrivées massives, par petits groupes ou même individuellement quittent des endroits où la vie est devenue impossible. Le phénomène est toujours différent et toujours le même dans le fond, pareil à ce qu'on a connu pendant le $\mathrm{XX}^{\mathrm{e}}$ siècle et même avant. Avec la mondialisation, qui, pour reprendre un concept de Michel Foucault, peut être considérée comme une mondialisation de la "société de contrôle", il y a une croissance statistique de la mobilité, mais de manière très inégale.

Pourtant, je pencherais volontiers pour une manière optimiste de dire les choses : simplement constater que nous sommes aujourd'hui dans un vaste processus de repeuple-

Les pays riches voudraient profiter de ce qu'il y a de mieux dans la mondialisation, à savoir le fait d'être mobile, tout en reproduisant une inégalité terrible, avec la mise à l'écart du Sud. ment du monde. Beaucoup de choses sont en train de se transformer pour des raisons politiques, écologiques et économiques et cela provoque des mobilités qui sont parfois très fortes. La planète se recompose et on évoque des centaines de millions de personnes déplacées d'ici cinquante ans. Dans ce cas, on aurait presque envie de dire : "accompagnons ce mouvement".

\section{H\&M : Cela demande une forte évolution des mentalités, car toutes les socié- tés privilégient la stabilité et la fixation.}

Certes, mais ces sociétés privilégient aussi la mobilité pour leurs classes dirigeantes, pour toutes ces populations qui peuvent apporter des avantages en termes démographiques ou de compétences. Maintenant, on commence à voir la Terre comme une seule entité vivante et on s'aperçoit qu'il y a des disparités de plus en plus grandes. Dans le débat sur les migrations, on assiste au retour de la question Nord/Sud et des inégalités. Les pays riches voudraient profiter de ce qu'il y a de mieux dans la mondialisation, à savoir le fait d'être mobile, tout en reproduisant une inégalité terrible, avec la mise à l'écart du Sud.

\section{H\&M : Tout ne se joue pas dans une dynamique Nord/Sud, il suffit d'observer les mobilités interrégionales, Sud/Sud.}

Effectivement, les personnes se déplacent beaucoup en Afrique ; on le voit notamment avec le phénomène des réfugiés. Les diasporas balayent tous les continents 
et ne se situent pas dans cette dynamique Nord/Sud, mais la traversent. Cette réalité des diasporas est importante à situer, surtout d'un point de vue démographique. On peut y voir à la fois une solution et un problème. Certains y voient en effet une solution, car dans l'exil elles maintiennent un lien, c'est un peu comme un "chez soi" qui se déplace dans le monde.

Par exemple, en Afrique, des commerçants hausa ont organisé des réseaux commerciaux en diaspora, qui servent à la fois de cadre commercial et géographique de circulation de personnes venant d'une même région. Avec le temps, cela se transforme beaucoup, et on ne peut pas parler uniquement d'un état diasporique, même si la diaspora hausa est très connue. Il y a des membres très ancrés localement, avec une "hausaisation" de réseaux qui se sont énormément élargis.

L'écrivain somalien Nuruddin Farah a publié un ouvrage à l'issue d'une enquête qu'il a menée en Europe sur l'installation des Somaliens (Hier, demain. Récits de la diaspora). Il observe qu'il y a des endroits où la diaspora fonctionne et d'autres où elle ne marche pas. Par exemple, en Suisse, les Somaliens restent dans des centres d'accueil et de contrôle où rien ne se passe. En revanche, en Italie, les hommes n'arrêtent pas de se rappeler de leur position clanique et de leur vie sociale en Somalie, étant très liés les uns aux autres - lien qui n'est pas forcément très efficace au niveau économique. Ancrées davantage dans la nécessité de travailler et de pourvoir aux besoins de la famille, les femmes s'occupent du noyau familial et se moquent profondément de la diaspora

Ainsi, en regardant dans le détail ce que, dans le milieu des sciences sociales, l'on appelle une "diaspora", il apparaît que ce concept fortement connoté ne permet pas toujours de rendre compte de la réalité des personnes éparpillées. Quelques grandes diasporas font qu'on surestime parfois l'efficacité de la diaspora par rapport à d'autres formes de migration. Or il n'y a pas forcément une stratégie délibérée dans le choix d'une localité d'installation. L'idée de la diaspora, définie comme "je transporte mon lieu", se diffuse et se transforme dans le monde, mais coïncide à une vision des élites.

\section{H\&M : En Afrique, ces mouvements de réfugiés prennent-ils des formes diasporiques?}

Le cas des Somaliens est assez clair à ce propos. J'ai travaillé sur les camps de Dadaab, qui se trouvent au Kenya près de la frontière somalienne. On y constate un cas d'arrangement et de combinaison de facteurs locaux très importants avec des réseaux plus élargis. Des réseaux triangulaires se forment entre les personnes qui sont restées en Somalie, ceux qui sont installés à Dadaab dans les camps et enfin ceux qui sont installés au Kenya, dans un quartier somali à Nairobi. Ces der- 
niers ne sont donc pas des réfugiés, car c'est un quartier, et non un camp. En effet, en Afrique, on est réfugié lorsque l'on décide de partir dans un camp. On est alors comptabilisé et pris en charge en tant que tel.

\section{H\&M : Mais les chiffres sont-ils fiables ? Sont-ils produits par les organisations intemationales, les Etats, les ONG ?}

Pour donner des chiffres, il faut d'abord produire des catégories. Or, le problème des contrôles de population, que ce soit au Kenya ou entre Guinée, Liberia et Sierra Leone, se pose d'abord lorsque des personnes se présentent à la frontière. L'attribution du statut de réfugié prima facies a été ajouté en 1967 au dispositif de Genève de 1951 pour caractériser ces nouvelles situations politiques. En 1951, la Convention de Genève naît à la sortie de la Seconde Guerre mondiale, en pleine guerre froide et concerne tous ceux qui viennent de l'Est après avoir échappé à un système politique oppressif. Tout persécuté est appelé réfugié politique, pour n'importe quelle raison et dans une acception assez large.

En 1967 a été ajouté le statut prima facies : on constate en effet que, dans un contexte donné, des populations passent la frontière et s'installent de l'autre 
côté. À ce moment, des organisations humanitaires créent une première prise en charge. Dans un deuxième temps, soit les réfugiés sont installés dans des camps apprêtés par le HCR à au moins $50 \mathrm{~km}$ de la frontière, soit ils partent d'euxmêmes et, dans ce cas-là, on considère qu'ils se débrouillent et ne sont plus comptabilisés comme réfugiés, ni ne peuvent bénéficier des aides fournies pour favoriser l'installation.

Dans des contextes violents très identifiés, ne sont reconnus comme réfugiés que ceux qui sont installés dans un camp. La preuve étant la carte du PAM (programme alimentaire mondial), qui agit comme une sorte de "carte d'identité" et atteste que la per-

En grande partie, aujourd'hui, l'Afrique entre dans

la mondialisation

"par le bas" et dans

des contextes de violence. sonne est bénéficiaire de la ration alimentaire dans un camp déterminé. Pour circuler, cette personne aura besoin d'une dérogation pour déjà sortir du camp. Parfois, ceux qui désirent rester en dehors du camp pendant une période plus longue donnent un peu d'argent aux policiers pour qu'ils les laissent circuler. Entre la Somalie et le Kenya des formes d'organisation de réseaux familiaux et sociaux se créent entre les personnes installées dans le quartier somali de Nairobi (commerçants travaillant dans le secteur informel), ceux qui sont restés dans les camps et peuvent sortir ou recevoir de l'argent venant des communautés du Kenya et enfin ceux qui sont restés à Mogadiscio et peuvent circuler entre Nairobi et les camps. Il ne faut pas oublier non plus ceux qui, installés à Dadaab, reçoivent de l'argent venant de membres de la famille qui ont migré en Europe ou aux ÉtatsUnis. Dadaab, qui était au début un petit village, s'est donc considérablement agrandi. On y trouve même des petites banques qui sont des correspondantes de Western Union, où les réfugiés vont chercher l'argent qu'ils reçoivent.

On trouve ceux qui restent - les déplacés internes -, ceux qui migrent à Nairobi avec un statut incertain, ceux qui se réfugient dans les camps et ceux qui, installés en Europe ou ailleurs, vont être pour la plupart considérés comme des migrants économiques, car on leur conteste la preuve de leur situation de réfugiés. Il y a donc autant de situations différentes que de formes de catégories selon les organisations internationales ou les États.

\section{H\&M : Est-ce que ces mouvements sont plutôt constitués par des familles ? Que peut-on dire en termes de genre, d'âge, etc. ?}

Cela dépend des contextes. Mais faisant référence à des situations de guerre ou de violence vues en Guinée ou en Zambie, tous les récits font état de petits groupes 
qui souvent se décomposent, entre le moment où tout le monde s'échappe et le long stationnement dans les camps. En Guinée par exemple, on trouve des réfugiés libériens ou sierra-léonais, avec des configurations familiales complètement éclatées. Les camps favorisent aussi des recompositions familiales rapides, on s'y marie beaucoup et on y fait beaucoup d'enfants.

Les hommes sont inclus dans les groupes armés d'un côté comme de l'autre, et éventuellement choisissent de ne pas s'installer dans les camps où il est impossible de travailler. En général, on trouve dans les camps des bouts de familles, femmes et enfants en bas âge, mais aussi des fratries ou des "pseudo fratries" d'adolescents qui ont subi des violences et ont été séparés de leurs parents, et encore qui ont fui avant ou après avoir été enrôlés de force. Les camps connaissent un fort déséquilibre démographique, qui n'est pas forcement toujours le même. À Kakuma au Kenya, par exemple, il y avait énormément de jeunes hommes âgés entre 20 et 25 ans, soit un ratio de 180 hommes pour 100 femmes, avec bien sûr tous les problèmes de rapts de femmes et de conflits que cela peut engendrer. Ils avaient fui le conflit au Soudan et les recrutements des groupes armés. En Guinée, j'ai vu aussi beaucoup de "fratries" de jeunes qui avaient été enrôlés. La longue durée des conflits et l'installation de dispositifs humanitaires internationaux créent un réseau entre les camps. Les réfugiés savent d'un endroit à l'autre où retrouver des membres disparus de la famille. Une scène typique des camps, au moment de l'arrivée de camions de réfugiés dans les zones de transit, c'est l'attroupement de ceux qui viennent chercher des personnes ou des informations sur tel village ou telle ville. Les réfugiés qui sont installés depuis deux ou trois ans dans un camp savent très bien ce qu'est devenu un proche qu'ils avaient perdu de vue depuis plus de cinq ans !

\section{H\&M : Est-on amené à vivre longtemps dans les camps puisque, par défini- tion, ils sont temporaires?}

Voilà le grand paradoxe. Il y a une forme de cristallisation des camps. Tout le monde a désormais pris conscience de la durée des camps et de ce qu'elle veut dire, en terme de précarité. Cette situation devient vite invivable pour ces personnes, car elle se prolonge au-delà de l'urgence immédiate à laquelle le dispositif humanitaire peut répondre. Après quelques semaines de gestion de l'urgence immédiate, due à un conflit ou à une situation médicale qui demande le regroupement des personnes, celles-ci s'installent dans la durée et toute la grosse machine humanitaire se met en place. Alors, ni les humanitaires ni les réfugiés n'ont envie que cette situation cesse. Le dispositif tend à s'autoreproduire. En Afrique, beaucoup de villes ont commencé par un campement. 


\section{H\&M : Économiquement, est-ce qu'un camp peut produire de la richesse ?}

Cela dépend de la période et du camp en question. Sur le plan historique, un camp est une forme de création d'une nouvelle ville, via des arrivées, la création de commerces, de structures de pouvoir et d'aménagement de l'espace. Actuellement, on a des dispositifs très artificiels au départ et très contrôlés ensuite : ce mouvement naturel de campement peut devenir une ville, mais, pour des raisons géopolitiques, économiques et politiques, ce type de développement est proposé tout en étant interdit. Tout ce qui ressemble à la vie qui reprend est interdit. C'est le cas de la revente de la ration alimentaire. Quoique éthiquement discutable, cela fait partie de cette reprise de la vie sociale et économique. La ration alimentaire ne recouvre pas les besoins réels. On est obligé de revendre une partie de féculent afin de se procurer des légumes ou des protéines.

\section{H\&M : Est-ce que les autonités locales sont capables de prendre le relais et de proposer une autre forme de gouvernance des camps?}

On peut le dire comme cela, mais il faudrait voir au cas par cas. C'est toujours un sujet de polémique et de débat entre les organisations internationales et les pouvoirs locaux. Je m'étonne de voir le HCR - grande organisation qui a le mandat de l'ONU de gérer tous ces phénomènes - accepter trop facilement la mise en camp des réfugiés alors qu'il serait possible, compte tenu de son origine onusienne et de son pouvoir politique et économique, de négocier avec les gouvernements africains pour passer à une assistance individuelle plutôt qu'à la mise en camp. Il n'y a rien de pire que d'être mis durablement dans un camp, dans des lieux choisis à l'écart de la vie réelle. Des situations de conflit très violent conduisent parfois à l'intervention de la police. Ce fut le cas face à la demande de réfugiés libériens en Guinée auxquels le HCR opposait le fait que, tant qu'ils n'accepteraient pas d'aller en camp, ils n'auraient droit à rien, sachant que les camps en question étaient à plus de $600 \mathrm{~km}$ de la frontière, en pleine Guinée forestière.

\section{H\&M : Est-ce que ces autorités locales peuvent mettre en place des poli- tiques d'accueil pour les réfugiés, qui seraient adaptées aux réalités des individus et aux contextes?}

Les guerres ou l'état d'appauvrissement politique et économique de certains pays en Afrique relèvent de la solidarité et de la responsabilité internationale. L'ONU crée des camps via le HCR, mais elle pourrait aider autrement des réfugiés ou des exilés qui sont obligés de partir ou "choisissent" de le faire dans un contexte devenu sans issue. Cette responsabilité internationale est très partiellement reconnue, alors qu'elle pourrait aussi se traduire par des prises en charge sur des projets collectifs ou individuels avec les acteurs locaux. 
Plusieurs anecdotes permettent de voir cette ambiguïté. Un représentant du HCR s'est fait agresser à Conakry par un groupe de réfugiés libériens, car il refusait de leur donner une assistance au titre de réfugié, faute de s'installer dans un camp. Ensuite, la police guinéenne a décidé d'effectuer des rafles pour envoyer ces personnes dans les camps, et le HCR a affirmé ne plus être responsable pour les personnes qui étaient encore à Conakry. Le poids politique et économique de l'ONU n'est pas comparable à celui de la Guinée, qui doit être en $169^{\circ}$ position de l'IDH (indice de développement humain). C'est le même HCR qui, en France, propose d'aller chercher dans les environs de Calais les demandeurs d'asile qui attendent de passer en Angleterre, pour les "aider" à rentrer chez eux. Cela correspond aussi à une forme d'adhésion à des politiques de contrôle des migrations et on peut se demander si le HCR n'a pas ainsi abandonné sa mission de protection telle qu'elle était formalisée en 1951.

Un autre événement s'est déroulé dans les camps installés en forêt guinéenne. Les représentants du HCR de Genève sont arrivés avec la bonne nouvelle de 4000 postes de réinstallations dans plusieurs pays tiers (Norvège, États-Unis, Australie), mais se sont refusés de sélectionner les réfugiés. On a donc demandé aux ONG de 
faire le tri, alors que c'est de la responsabilité du HCR d'identifier les modalités de sélection. Il demande aux ONG de faire le "sale boulot" et, si une partie des ONG refusent, d'autres acceptent ce rôle et entretiennent auprès des réfugiés une forme d'attente et d'espoir, tout en disant qu'il y aura une stricte sélection.

Dernier exemple, une réinstallation massive en pays tiers a été décidée pour un groupe de réfugiés installés à Dadaab et considéré comme ethniquement minoritaire en Somalie, hors caste. Dans les camps, ce groupe a demandé que sa diversité soit reconnue. Le HCR a donc décidé qu'une réinstallation serait réservée à 10000 membres de cette communauté aux États-Unis. Après cette annonce, plusieurs Somalis ont essayé de se faire passer pour des membres de cette communauté et le HCR s'est donc plaint des "tricheurs". Pourtant, cela est prévisible lorsqu'on met en place de telles différences de traitement de la sorte.

De plus, on part de présupposés erronés comme le fait de dire que les réfugiés africains veulent tous aller en Europe ; ce qui est totalement faux. Cela est typique d'une situation de domination : on crée à la fois de la fascination pour les pays de destination et de l'interdiction en limitant l'accès. Tout ce système est à repenser.

\section{H\&M : Très schématiquement, peut-on dessiner une sorte de cartographie de ces mouvements de réfugiés sur le continent africain ?}

C'est une question très compliquée, et la réponse évolue. Dans les années quatrevingt-dix et jusqu'en 2004, une zone très importante se situait en Afrique de l'Ouest, le long du fleuve Mano (Guinée, ouest de la Côte d'Ivoire, Libéria, Sierra Leone). Il y a eu aussi, et même depuis les années soixante-dix, un foyer en Angola, au Congo et en Zambie, avec des camps d'Angolais qui subsistent encore en Zambie. Beaucoup de camps accueillaient des déplacés qui n'ont pas franchi la frontière et ne relèvent donc pas d'une organisation internationale.

Cependant, depuis 2006, le HCR a le mandat non pas de la gestion des déplacés, mais des camps de déplacés. Cela montre bien sa spécialisation dans le domaine de la gestion. Il y a eu des discussions internes à l'ONU, entre OCHA - le bureau de l'ONU pour les affaires humanitaires - et le HCR, et c'est finalement ce dernier qui a obtenu le mandat pour les "abris d'urgence" et les camps. On en a donc conclu que cela incluait les camps de déplacés.

Aujourd'hui, on trouve des déplacés internes au Darfour, et des réfugiés dans la zone entre le Soudan, le Tchad, l'Ouganda, ou encore au Kenya qui accueille des Soudanais ou des Somaliens, ainsi que l'est de la RDC, avec de nombreux déplacés. Cependant, dans les zones frontalières, il est toujours difficile de savoir si les personnes ont véritablement traversé la frontière. Récemment, les épisodes de violence en Afrique du Sud contre les Zimbabwéens ont créé une situation particulière. 
Pour rester en Afrique, il y a beaucoup plus de déplacements de population pour cause de conflit d'un pays à l'autre, et souvent entre pays voisins tout simplement. Les plus pauvres ne partent pas ou demeurent des déplacés internes; ceux qui le peuvent partent ensuite dans le pays d'à côté et d'autres encore développent des stratégies différentes pour aller plus loin.

La thèse que je défends est qu'il s'agit pour l'Afrique d'une manière bien étrange d'entrer dans la mondialisation. Les personnes découvrent le monde avec les organisations internationales, les personnels humanitaires, les armées onusiennes, la culture de l'humanitaire, la conduite des projets, les changements de mode de vie, d'habitat ; ce qui entraîne un fort changement culturel sur le fond. En grande partie, aujourd'hui, l'Afrique entre dans la mondialisation "par le bas" et dans des contextes de violence. 